\title{
An olive branch to two theories
}

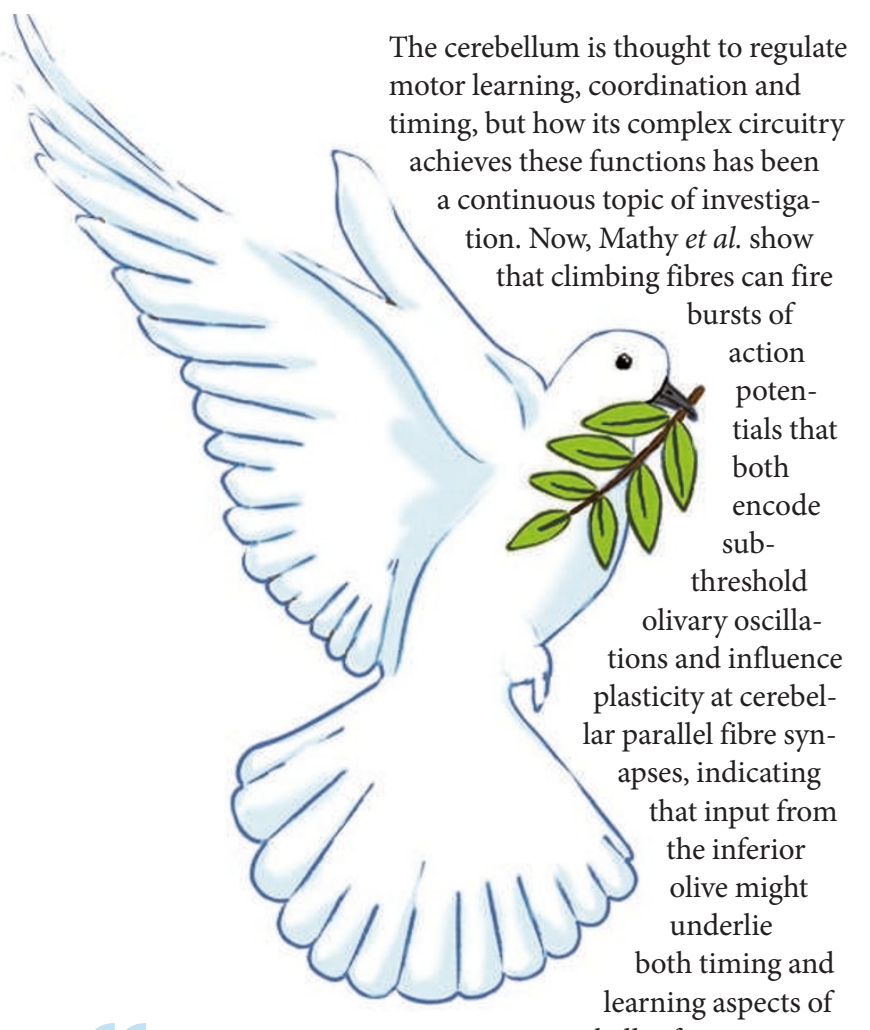

cerebellar function.

...climbing

fibre bursts

influence

Purkinje cell

firing patterns

and probably

contribute to

motor timing.
The authors next determined whether the characteristic spontaneous subthreshold membrane potential oscillations in olivary neurons can influence axonal bursts. They generated oscillations by injecting sinusoidal current and then triggering action potentials through synaptic stimulation at different phases of the oscillatory period. The number of spikes in each axonal burst depended on the phase of the oscillatory cycle at which the synaptic input occurred, whereas the timing between spikes in a burst was highly reproducible between trials.

To establish whether the axonal bursts in climbing fibres are transmitted to their Purkinje cell targets, the authors recorded excitatory postsynaptic currents (EPSCs) from Purkinje cells in vivo. Spontaneous climbing fibre-mediated EPSCs could be measured in Purkinje cells and, in half of the recordings, the initial EPSC was followed by a burst of up to three EPSCs. The timing of the EPSCs in a burst was highly reproducible and was similar to the timing of olivary axon burst spikes, suggesting that bursts were transmitted from climbing fibres to Purkinje cells.

To investigate the consequences of this burst transmission the authors recorded from Purkinje cell somas and dendrites while climbing fibres were stimulated in brain slices. The number of spikes in the characteristic 'complex-spike' response recorded at the Purkinje cell somas correlated with the number of climbing fibre stimuli, with higher numbers of stimuli also triggering more dendritic $\mathrm{Ca}^{2+}$ spikes. This indicates that climbing fibre bursts influence Purkinje cell firing patterns and probably contribute to motor timing.

Synapses between parallel fibres and Purkinje cells can undergo longterm depression when parallel fibre input coincides with climbing fibre input, but do climbing fibre bursts have a particular role in this? The authors paired parallel fibre stimulation with either a single climbing fibre stimulus or a burst of stimuli, and found that only the latter combination reliably induced long-term depression. In addition, climbing fibre bursts enhanced shortterm plasticity at these synapses, with the number of stimuli in the burst correlating with the degree of parallel fibre suppression.

The finding that climbing fibres can fire graded bursts of action potentials suggests that inferior olive neuron signals are more informative than the all-or-nothing output that has traditionally been attributed to them. Moreover, the data show that these bursts might encode both a timing signal and a learning signal, thus integrating two major theories of climbing fibre function.

Leonie Welberg

ORIGINAL RESEARCH PAPER Mathy, A. et al. Encoding of oscillations by axonal bursts in inferior olive neurons. Neuron 62, 388-399 (2009) 